\title{
Chemsketch Study of Phenobarbital: An Antiepileptic Drug
}

\section{Katanguru Laxmi}

Department of Chemistry, Chaitanya Bharathi Institute of Technology (CBIT), Hyderabad, India

\section{Email address:}

katangurulaxmi@gmail.com

\section{To cite this article:}

Katanguru Laxmi. Chemsketch Study of Phenobarbital: An Antiepileptic Drug. International Journal of Computational and Theoretical Chemistry. Vol. 5, No. 3, 2017, pp. 25-29. doi: 10.11648/j.ijctc.20170503.11

Received: March 24, 2017; Accepted: April 19, 2017; Published: May 27, 2017

\begin{abstract}
Phenobarbital belongs to the class of medications called barbiturates. It is used to treat insomnia (difficulty sleeping) and as a sedative to relieve the symptoms of anxiety or tension. It is also used for the control of certain types of seizures. It works by slowing down the brain and nervous system. Phenobarbital is a barbiturate, nonselective central nervous system depressant which is primarily used as a sedative hypnotic and also as an anticonvulsant in subhypnotic doses.
\end{abstract}

Keywords: Phenobarbital, An Anticonvulsant and CNS Activity, ACD/ChemSketch, ACD/3D Viewer

\section{Introduction}

Phenobarbital belongs to a class of drugs known as barbiturate anticonvulsants/hypnotics [1]- [3]. Barbiturates are substituted pyrimidine derivatives in which the basic structure common to these drugs is barbituric acid, a substance which has no central nervous system (CNS) activity. CNS activity is obtained by substituting alkyl, alkenyl, or aryl groups on the pyrimidine ring. Phenobarbital is chemically Designated as 5-Ethyl -5-phenyl barbituric acid. Phenobarbital (Figure 1) works by controlling the abnormal electrical activity in the brain that occurs during a seizure. This medication is also used for a short time (usually no more than 2 weeks) to help calm you or help you sleep during periods of anxiety [4]- [6].
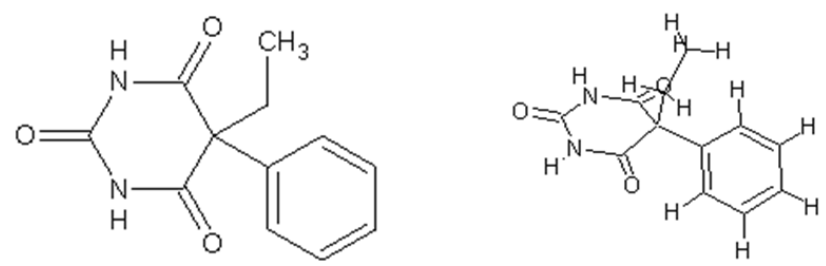

Figure 1. structure of Phenobarbital.

\subsection{Hypothetical Study of Phenobarbital}

ACD/Labs [7]- [9] provides a Chemical Naming Service that will use our powerful software to quickly and accurately name compounds. ACD/Name features enhanced naming capabilities that allow you to generate systematic names according to both IUPAC and CAS Index nomenclature rules, in addition to generating chemical structures from names.

Molecular Formula of Phenobarbital is $\mathrm{C}_{12} \mathrm{H}_{12} \mathrm{~N}_{2} \mathrm{O}_{3}$ and its Molecular Weight is 232.24.

Name of structure 5-ethyl-5-phenylpyrimidine-2,4,6 $(1 H, 3 H, 5 H)$-trione.

InChI name of Phenobarbital.

$\mathrm{InChI}=1 \mathrm{~S} / \mathrm{C} 12 \mathrm{H} 12 \mathrm{~N} 2 \mathrm{O} 3 / \mathrm{c} 1-2-12(8-6-4-3-5-7-8) 9(15) 13-$ 11(17)14-10(12)16/h3-7H,2H2,1H3, (H2,13,14,15,16,17).

The IUPAC International Chemical Identifier, InChI, was developed as an accurate code to denote the exact configuration of a molecule. With the InChI code, a molecule is unambiguously described by means of its stereochemistry, tautomeric, isotope and electronic charge information [10][11]. The IUPAC International Chemical Identifier ( $\mathrm{InChI}$ ) is a textual identifier for chemical substances, designed to provide a standard and human-readable way to encode molecular information and to facilitate the search for such information in databases and on the web. Initially developed by IUPAC and NIST from 2000 to 2005 , the format and algorithms are non-proprietary. The current version is 1.04 and was released in September 2011. Prior to 1.04, the software was freely available under the open source LGPL license, but it now uses a custom license called IUPAC-InChI Trust License.

An InChI label is generated by converting an input 
chemical structure (in the form of a 'connectiontable') to a unique and predictable set of ASCII characters. The InChI notation is a way of representing chemical compounds in a manner that does not depend on the way of drawing. InChI procedures were developed under IUPAC project during the period 2000-2004. The technical development was carried out primarily at the US National Institute of Standards and Technology (NIST).

\section{2. 'Auxiliary Information' (AuxInfo) of Phenobarbital}

The InChI Software output complements the Identifier itself with a range of additional information. This includes warnings and errors messages, as well as a specific 'Auxiliary Information' (AuxInfo) string.

AuxInfo $^{15}$ contains, in particular, atom non-stereo equivalence information, mapping input atom positions to output positions, and 'reversibility' information for redrawing the structure. AuxInfo is generated by the inchi-1 executable by default (this behavior may be turned off by using the switch AuxNone). Note that the AuxInfo string itself is a valid input for the inchi-1 generator and may be used to regenerate the source structure.

AuxInfo=1/1/N:13,12,5,4,6,3,7,2,8,9,16,1,14,15,10,11,17/ $\mathrm{E}:(4,5)(6,7)(9,10)(13,14)(15,16) / \mathrm{rA}: 17 \mathrm{CCCCCCCCCOOCC}$ NNCO/rB:s1;s2;d3;s4;d5;d2s6;s1;s1;d8;d9;s1;s12;s8;s9;s14s 15;d16;/rC:536,-357,0;691,-268,0;845,-357,0;1000,268,$0 ; 1000,-89,0 ; 845,0,0 ; 691,-89,0 ; 447,-203,0 ; 447,-$ 512,$0 ; 536,-48,0 ; 536,-667,0 ; 691,-447,0 ; 691,-625,0 ; 268,-$ 203,$0 ; 268,-512,0 ; 179,-357,0 ; 0,-357,0$.

\subsection{E Molecules}

E Molecules is a search engine for chemical molecules. The system was founded by scientists for scientists in 2005 with one goal in mind: reduce drug discovery timelines through improved efficiencies. E molecules of Phenobarbital is given in figure 2 .

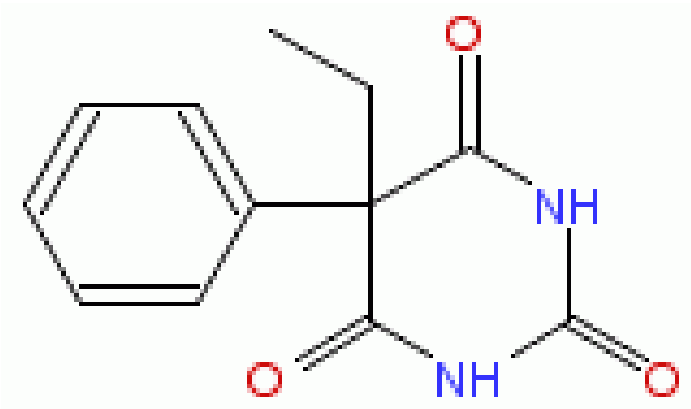

Figure 2. E molecules of Phenobarbital.

e molecules of Phenobarbital - Properties

$\mathrm{M} \mathrm{Wt} 232,235$

$M F \mathrm{C}_{12} \mathrm{H}_{12} \mathrm{~N}_{2} \mathrm{O}_{3}$

SMILES: $\operatorname{CCC} 1(\mathrm{C}(=\mathrm{O}) \mathrm{NC}(=\mathrm{O}) \mathrm{NC} 1=\mathrm{O}) \mathrm{c} 1 \mathrm{ccccc} 1$

CAS: $50-06-6$

CAS: $73738-05-3$

Name: 5-Ethyl-5-phenyl-pyrimidine-2,4,6-trione

\subsection{Smiles Notation of Phenobarbital}

The Simplified Molecular-Input Line-Entry System (SMILES) encodes the connectivity of atoms within a compound in an ASCII string, but does not offer 2D or 3D coordinates for molecules.

The simplified molecular-input line-entry system (SMILES) [12]- [13] is a specification in form of a line notation for describing the structure of chemical species using short ASCII strings. SMILES strings can be imported by most molecule editors for conversion back into twodimensional drawings or three-dimensional models of the molecules. The original SMILES specification was initiated in the 1980s. It has since been modified and extended.Smiles notation of

Phenobarbital $\operatorname{CCC} 1(\mathrm{C}(=\mathrm{O}) \mathrm{NC}(=\mathrm{O}) \mathrm{NC} 1=\mathrm{O}) \mathrm{c} 1 \mathrm{ccccc} 1$.

\subsection{Chemspider of Phenobarbital}

ChemSpider is a database of chemicals which is owned by the Royal Society of Chemistry. ChemSpider [14]- [18] is a free chemical structure database providing fast text and structure search access to over 47 million structures from hundreds of data sources. Chemspider structure of Phenobarbital is given in figure 3 .

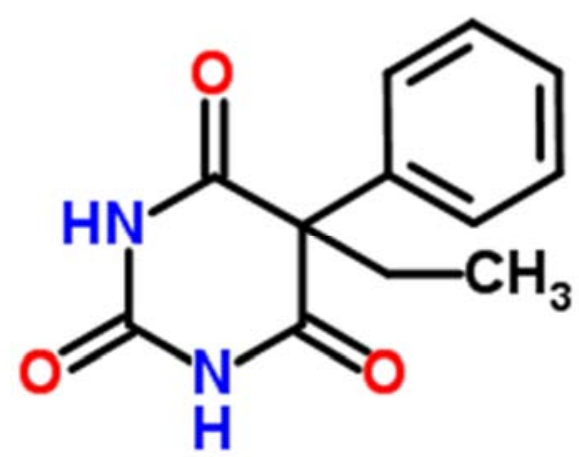

Figure 3. Chemspider structure of Phenobarbital.

Chemspider data of Phenobarbital

a) Molecular Formula $\mathrm{C}_{12} \mathrm{H}_{12} \mathrm{~N}_{2} \mathrm{O}_{3}$

b) Average mass $232.235 \mathrm{Da}$

c) Monoisotopic mass $232.084793 \mathrm{Da}$

d) Chemspider ID 4599

5-Ethyl-5-phenyl-2,4,6(1H,3H,5H)-pyrimidinetrione [ACD/IUPAC Name]

2,4,6(1H,3H,5H)-Pyrimidinetrione, 5-ethyl-5-phenyl[ACD/Index Name]

In addition to the drawing capabilities, ACD/ ChemSketch offers predictions of numerous properties for compounds. These include prediction of Molecular formula, Formula weight, Composition, Molar refractivity, Molar volume, Parachor, Index of refraction, Surface tension, Density, Dielectric constant, Polarizability, Monoisotopic, nominal, and average mass.

In this paper, the simple means to calculate these properties and the calculation algorithms are briefly described. 


\section{Results and Discussions}

\section{Algorithms for Calculating Properties}

At the heart of the additive-constitutive calculation algorithm of all physicochemical properties in ACD/ChemSketch lies the presumption that these properties can be estimated using additive atomic or group increments [19]. Apart from molecular weight (MW), which is trivial to calculate, the algorithms may be divided into three general groups:

\subsection{Basic Macroscopic Properties: Molar Volume (MV), Molar Refractivity (MR), and Parachor (Pr)}

\subsubsection{Molar Volume, MV}

By definition,

$$
\mathrm{MV}=\mathrm{MW} / \mathrm{d}
$$

ACD/ChemSketch calculates molar volume from additive increments. The additive atomic increments were obtained using a database of density and calculated MW.

\subsubsection{Molar Refractivity, $M R$}

The Lorentz-Lorenz equation relates refractive index, density, and refractive index

$$
\mathrm{MR}=\mathrm{n}^{2}-1 / \mathrm{n}^{2}-2 . \mathrm{MW} / \mathrm{d}
$$

ACD/ChemSketch calculates molar refractivity from additive increments. The additive atomic increments were obtained using a database of density, refractive index, and calculated MW.

\subsubsection{Parachor, $P r$}

By definition,

$$
\mathrm{P}_{\gamma}=[\mathrm{MW} / \mathrm{d}] \gamma^{1 / 4}
$$

$\mathrm{ACD} / \mathrm{ChemSketch}$ calculates the parachor from additive increments. The additive atomic increments were obtained using a database of density, surface tension, and calculated MW.

\subsection{Derived Macroscopic Properties: Density (d), Refractive Index (n), and Surface Tension ( $\gamma$ )}

\subsubsection{Density, $d$}

By definition,

$$
\mathrm{d}=\mathrm{MW} / \mathrm{MV}
$$

ACD/ChemSketch calculates the density from MW and the calculated molar volume (see above).

\subsubsection{Refractive Index, $n$}

By the Lorentz-Lorenz equation,

$$
\mathrm{n}=\sqrt{ } 2 \cdot \mathrm{MR}+\mathrm{MV} / \mathrm{MV}-\mathrm{MR}
$$

$\mathrm{ACD} / \mathrm{Chem}$ Sketch calculates the refractive index from the molar volume and molar refractivity, both of which are calculated as above.

\subsubsection{Surface Tension, $\gamma$}

By definition,

$$
\gamma=\left[\mathrm{P}_{\gamma} / \mathrm{MV}\right]^{4}
$$

$\mathrm{ACD} /$ ChemSketch calculates the surface tension from calculated MV (see above) and calculated

$\operatorname{Pr}$ (see above).

\subsection{The Dielectric Constant $\varepsilon$ (Permittivity)}

Basic macroscopic properties such as molar volume (MV), molar refractivity (MR), and the parachor $(\mathrm{Pr})$ are calculated first for the input structure. The atomic additive increments in such an algorithm depend on the bonds (single, double, aromatic, etc.) of this atom and on neighboring atoms. $\mathrm{ACD} / \mathrm{ChemSketch}$ rapidly analyzes the input structure to determine the class of each atom, i.e., whether it is cyclic, aromatic, aliphatic, etc. The prediction algorithms for density $(d)$, refractive index $(n)$ and surface tension $(\gamma)$ are founded on well-known physicochemical formula which can be found in literature on physicochemical properties of compounds.

They express $d, n$, and $\gamma$ as functions of MV, MR, or $\mathrm{P} r$. Once the MV, MR, or $\mathrm{Pr}$, have been predicted by additive means, it is straightforward to predict $d, n$, and $\gamma$ using these formula.

The determination of the additive-constitutive atomic increments for $\mathrm{MV}, \mathrm{MR}$, and $\mathrm{Pr}$ were obtained internally by ACD/Labs scientists using large experimental databases relating structure to density, refractive index, and surface tension. The MV, MR, and $\mathrm{Pr}$ were recalculated from $d, n$, and $\gamma$. These parameters are proprietary information of ACD/Labs, Inc. The prediction of the dielectric constant $\varepsilon$ (permittivity) resembles very closely the prediction of boiling point, which is a separate $\mathrm{ACD} / \mathrm{Labs}$ product available from $\mathrm{ACD} /$ ChemSketch. Senior scientists at ACD/Labs discovered an additive function, which relates the dielectric constant to other macroscopic properties which can be additively treated, such as MV. Once this relationship was discovered, the additive-constitutive atomic increments for this function were obtained using large databases consisting of molecular structures and their observed dielectric constants.

Using the function and estimated MV for the input structure, its dielectric constant can be quickly predicted.

By definition,

$$
\mathrm{f}(\varepsilon)=\mathrm{f}(\mathrm{MV}, \text { AdditiveFunction })
$$

$\mathrm{ACD} /$ ChemSketch calculates the dielectric constant from calculated MV (see above) and a proprietary empirical additive function.

\subsection{Polarizability}

This property is calculated from the Molar Refractivity (MR) as follows:

$$
\text { Polarizability }=0.3964308 \cdot \mathrm{MR}
$$

\subsection{Monoisotopic, Nominal and Average Mass}

Monoisotopic mass (Mmi) is the exact mass of the most abundant stable isotope that can occur naturally. 
Nominal Mass (Mn) is the sum of the approximated monoisotopic masses of the elements forming the structure.

Average Mass (Mav) is the calculated mass of a particle based on the atomic weights of the elements from which it is comp.

$\begin{array}{ll}\text { Molecular Formula: } & \mathrm{C}_{12} \mathrm{H}_{12} \mathrm{~N}_{2} \mathrm{O}_{3} \\ \text { Formula Weight: } & 232.23528 \\ & \mathrm{C}(62.06 \%) \mathrm{H}(5.21 \%) \mathrm{N}(12.06 \%) \\ \text { Composition: } & \mathrm{O}(20.67 \%) \\ \text { Molar Refractivity: } & 59.21 \pm 0.3 \mathrm{~cm} 3 \\ \text { Molar Volume: } & 188.1 \pm 3.0 \mathrm{~cm} 3 \\ \text { Parachor: } & 482.8 \pm 6.0 \mathrm{~cm} 3 \\ \text { Index of Refraction: } & 1.541 \pm 0.02 \\ \text { Surface Tension: } & 43.3 \pm 3.0 \text { dyne } / \mathrm{cm}^{3} \\ \text { Density: } & 1.233 \pm 0.06 \mathrm{~g} / \mathrm{cm}^{3} \\ \text { Dielectric Constant: } & \text { Not available } \\ \text { Polarizability: } & 23.47 \pm 0.510-24 \mathrm{~cm}^{3} \\ \text { RDBE: } & 8 \\ \text { Monoisotopic Mass: } & 232.084792 \mathrm{Da} \\ \text { Nominal Mass: } & 232 \mathrm{Da} \\ \text { Average Mass: } & 232.2353 \mathrm{Da} \\ \mathrm{M}^{+}: & 232.084244 \mathrm{Da} \\ \mathrm{M}^{-}: & 232.085341 \mathrm{Da} \\ {[\mathrm{M}+\mathrm{H}]^{+}:} & 233.092069 \mathrm{Da} \\ {[\mathrm{M}+\mathrm{H}]^{+}:} & 233.093166 \mathrm{Da} \\ {[\mathrm{M}-\mathrm{H}]^{+}:} & 231.076419 \mathrm{Da} \\ & \end{array}$

The partition constant, $P$, is a measure of the propensity of a neutral molecule to differentially dissolve in two immiscible phases, and serves as a quantitative descriptor of lipophilicity. The $\log P$ prediction model provides an estimate of the value of the octanol-water partitioning coefficient (also referred to as $\left.\mathrm{K}_{\mathrm{OW}}\right)$ as the $\operatorname{logarithmic}$ ratio $(\log P)$ from structure. $\mathrm{ACD} / \log P$ is used worldwide by chemists in various arms of chemical research, including some of the world's largest pharmaceutical companies (GlaxoSmithKline and Pfizer, to name just a few). Calculated $\log \mathrm{P}$ of Phenobarbital $=1.67+/-0.25$.

\section{3D Viewer -3D Optimised Forms of Phenobarbital}

$\mathrm{ACD} / 3 \mathrm{D}$ Viewer is a fast yet accurate $3 \mathrm{D}$ modeling and visualization program [20]. It is fully integrated with $\mathrm{ACD} /$ ChemSketch, allowing you to draw 2D structures and quickly obtain their $3 \mathrm{D}$ representations in a striking 16 color display. With ACD/3D Viewer you can:

a) Manipulate $3 \mathrm{D}$ models: move, $2 \mathrm{D}$ and $3 \mathrm{D}$ rotate, also at a fixed angle, resize, change styles, and colors;

b) Display a 3D structure as stick, ball-and-stick, spheres, or disks;

c) Add an overlay of small-dots at approximately the Van der Waals radius level to the solid 3D structure;

d) Measure and change bond lengths, bond angles, and torsion angles;

e) Optimize the structure using a 3D CHARMM-type of force field;

f) Switch from $3 \mathrm{D}$ to $2 \mathrm{D}$ display in the ChemSketch window at the click of a button;

g) Set the 3D structure to Auto-rotate, with or without changing the style of structure display;

h) Rotate and move selected atoms rather than entire structures.

i) Change and delete atoms, assign the center of rotation to an atom.

j) Create movies and save them as.GIF files;

k) View 3D structure in perspective; and

1) Export 3D models to other geometry optimization programs and use them as good starting configurations.

The ACD/3D Viewer, being powerfull modeling and visualization program, presents you with various styles of structure 3D representation. The 3D structure can be displayed as wireframe, sticks, sticks with dots, dots only, Ball and sticks, ball and sticks with dots, spacefills, disks, disks with dots forms. The various 3D forms of Phenobarbital are given in figures 4-8.

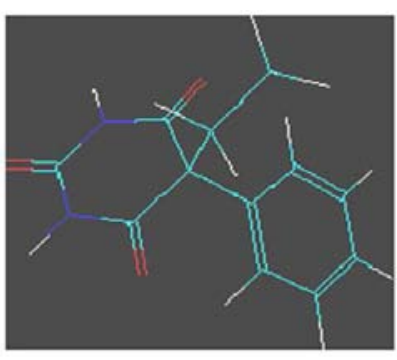

wireframe

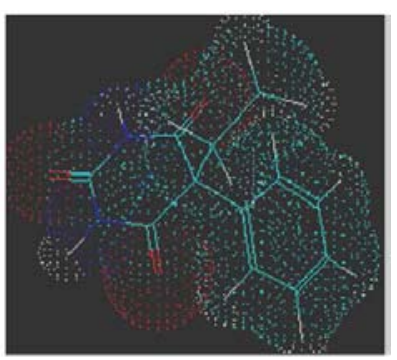

wireframe with dots
Figure 4. 3D optimized wireframe and wireframe with dots forms of Phenobarbital.

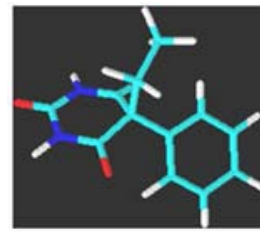

Sticks

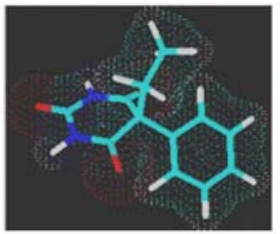

Sticks with dots

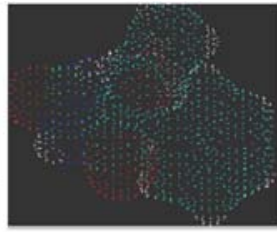

Dots only
Figure 5. 3D optimized Sticks, Sticks with dots and Dots only forms of Phenobarbital.

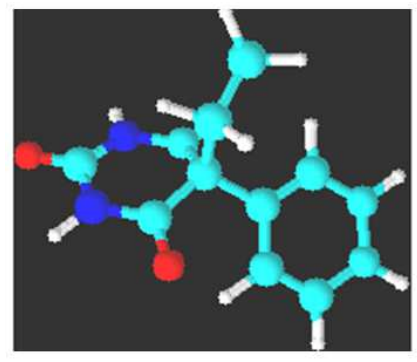

Ball and sticks

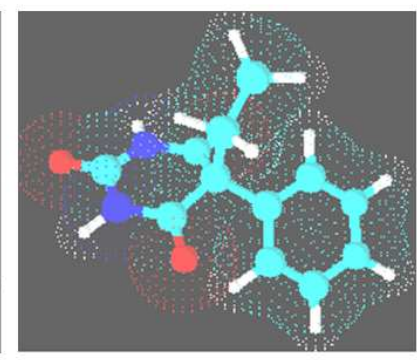

Ball and sticks with dots
Figure 6. $3 D$ optimized Ball and sticks form and Ball and sticks form with dots of Phenobarbital. 


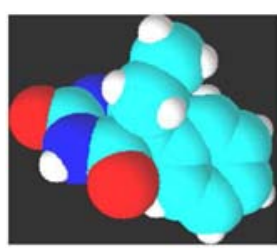

Space fill

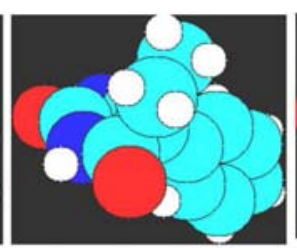

disks

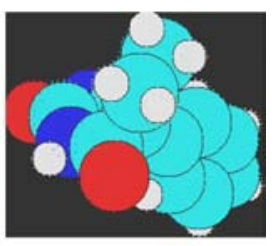

disks with dots
Figure 7. 3D optimized spacefill form, disks form and disks with dots forms of Phenobarbital.
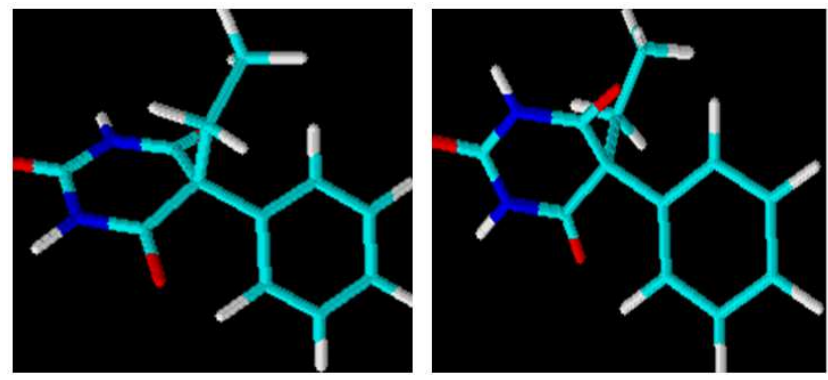

Figure 8. Mirror images of 3D optimized forms of Phenobarbital.

\section{Conclusion}

The chemsketch study of Phenobarbital is informative in understanding various structural and physicochemical aspects of the compound. The ACD/3D Viewer fully integrated with $\mathrm{ACD} /$ ChemSketch, helps in visualizing the various styles of structure 3D representation of Phenobarbital.

\section{References}

[1] Ilangaratne, NB; Mannakkara, NN; Bell, GS; Sander, JW (Dec 1, 2012). "Phenobarbital: missing in action." Bulletin of the World Health Organization 90(12): 871-871A. doi:10.2471/BLT.12.113183. PMID 23284189.

[2] Brodie, MJ; Kwan, P (December 2012). "Current position of phenobarbital in epilepsy and its future". Epilepsia. 53 Suppl 8: 40-6. doi:10.1111/epi.12027. PMID 23205961.

[3] "The epilepsies: the diagnosis and management of the epilepsies in adults and children in primary and secondary care NICE guidelines [CG137]". National Institute for Health and Care Excellence. January 2012.

[4] "Prescribing medicines in pregnancy database". Australian Government. 3 March 2014. Retrieved 22 April2014.

[5] Marc, Inman Nyack; Haverstraw, N.; Bilter, William. "Preparation of Phenobarbital (US2358072, 1944)" (PDF).Google Patents. Kay-Fries Chemicals, Inc. Retrieved30 October 2014.

[6] Http://www.deadiversion.usdoj.gov/schedules/orangebook/c_c s alpha.pdf page 1,7, 12, accessed 23.I.2016.
[7] "Chemsketch". Retrieved 21 August 2014.

[8] "Utilizando Chemsketch". Retrieved 21 August 2014.

[9] ACD/ChemSketch Freeware, version 11 (2006) Advanced Chemistry Development Inc., Toronto, ON, Canada, www.acdlabs.com.

[10] International Union of Pure and Applied Chemistry, Organic Chemistry Division Commission on Nomenclature of Organic Chemistry, Nomenclature of Organic Chemistry, Sections A, B, C, D, E, F, and H, 1979 Edition, J. Rigaudy and S. P. Klesney, eds.,Pergamon Press, Oxford, 1979, (Sections A, B, $\mathrm{C}, \mathrm{D}, \mathrm{E}$, and $\mathrm{F}$ are available).

[11] International Union of Pure and Applied Chemistry, Organic Chemistry Division, Commission on Nomenclature of Organic Chemistry (III.1), A Guide to IUPAC Nomenclature of Organic Compounds (Recommendations 1993), R. Panico, W.H. Powell and Jean-Claude Richer, eds., Blackwell Scientific Publications, Oxford, 1993.

[12] "SMILES Tutorial: What is SMILES?". U.S. Environmental Protection Agency. Retrieved 2012-09-23.

[13] National Center for Biotechnology Information (NCBI). PubChem Compound. (accessed May 12, 2012) PubChem Compound CID=183413 (Cephalostatin-1).

[14] Pence, H. E.; Williams, A. (2010). "ChemSpider: An Online Chemical Information Resource". Journal of Chemical Education 87 (11): 1123.doi:10.1021/ed100697w.

[15] Little, J. L.; Williams, A. J.; Pshenichnov, A.; Tkachenko, V. (2011). "Identification of "Known Unknowns" Utilizing Accurate Mass Data and ChemSpider". Journal of the American Society for Mass Spectrometry 23 (1): 179-85. doi: 10.1007/s13361-011-0265-y. PMID 22069037.

[16] "ChemSpider SyntheticPages". ChemSpider SyntheticPages. Royal Society of Chemistry. Retrieved26 June 2012.

[17] "ChemSpider and SyntheticPages support synthetic chemistry". RSC Publishing. Royal Society of Chemistry. 2010-02-05. Archived from the original on 26 July 2012. Retrieved 2012-06-26.

[18] Williams, A. J.; Harland, L.; Groth, P.; Pettifer, S.; Chichester, C.; Willighagen, E. L.; Evelo, C. T.; Blomberg, N.; Ecker, G.; Goble, C.; Mons, B. (2012). "Open PHACTS: Semantic interoperability for drug discovery". Drug Discovery Today 17 (21-22): 1188-1198.doi:10.1016/j.drudis.2012.05.016. PMID 22683805 .

[19] P. Wheeler, S. Hayward, M. Elyashberg, "182492-ComputerAssisted Structure Elucidation in Routine Analysis" Jan 26, 2016.

[20] ChemSketch Technical Note: Advanced Features and Functions, S. Myles et al. 